Draft Version OCtOBER 21, 2018

Preprint typeset using $\mathrm{L}_{\mathrm{A}} \mathrm{T}_{\mathrm{E}} \mathrm{X}$ style emulateapj v. 5/2/11

\title{
ALMA BAND 8 CONTINUUM EMISSION FROM ORION SOURCE I
}

\author{
Tomoya Hirota ${ }^{1,2}$, Masahiro N. Machida $^{3}$, Yuko Matsushita ${ }^{3}$, Kazuhito Motogi ${ }^{4,5}$, NaOko Matsumoto ${ }^{1,6}$, Mi $^{2}$ \\ Kyoung Kim ${ }^{7}$, Ross A. Burns ${ }^{8}$, \& Mareki Honma ${ }^{4,9}$ \\ Draft version October 21, 2018
}

\begin{abstract}
We have measured continuum flux densities of a high-mass protostar candidate, a radio source I in the Orion KL region (Orion Source I) using the Atacama Large Millimeter/Submillimeter Array (ALMA) at band 8 with an angular resolution of $0.1^{\prime \prime}$. The continuum emission at 430, 460, and $490 \mathrm{GHz}$ associated with Source I shows an elongated structure along the northwest-southeast direction perpendicular to the so-called low-velocity bipolar outflow. The deconvolved size of the continuum source, $90 \mathrm{au} \times 20 \mathrm{au}$, is consistent with those reported previously at other millimeter/submillimeter wavelength. The flux density can be well fitted to the optically thick black-body spectral energy distribution (SED), and the brightness temperature is evaluated to be 700-800 K. It is much lower than that in the case of proton-electron or $\mathrm{H}^{-}$free-free radiations. Our data are consistent with the latest ALMA results by Plambeck \& Wright (2016), in which the continuum emission have been proposed to arise from the edge-on circumstellar disk via thermal dust emission, unless the continuum source consists of an unresolved structure with the smaller beam filling factor.

Subject headings: ISM: individual objects (Orion KL) — radio continuum: stars — stars: formation — stars: individual (Orion Source I)
\end{abstract}

\section{INTRODUCTION}

The Orion Kleinmann-Low (KL; Kleinmann \& Low 1967) region is known to be the nearest site of highmass star-formation at a distance of 420 pc from the Sun (Menten et al. 2007; Kim et al. 2008). There are numbers of deeply embedded young stellar objects (YSOs) detected in X-ray, infrared, and radio wavelengths. In particular, high resolution radio interferometer observations have identified dust continuum sources, HiI regions, and radio jets driven by YSOs (e.g. Garay et al. 1987; Zapata et al. 2004; Rivilla et al. 2015; Forbrich et al. 2016).

Among a number of high-mass YSO candidates, a radio source I (Orion Source I) has the most outstanding characteristics. It has been thought to be a prototypical high-mass protostar candidate with a luminosity greater than $10^{4} L_{\odot}($ Menten \& Reid 1995). Source I is

\section{tomoya.hirota@nao.ac.jp}

${ }^{1}$ Mizusawa VLBI Observatory, National Astronomical Observatory of Japan, Osawa 2-21-1, Mitaka, Tokyo 181-8588, Japan

2 Department of Astronomical Sciences, SOKENDAI (The Graduate University for Advanced Studies), Osawa 2-21-1, Mitaka, Tokyo 181-8588, Japan

3 Department of Earth and Planetary Sciences, Faculty of Sciences, Kyushu University, Motooka 744, Nishi-ku, Fukuoka, Fukuoka 819-0395, Japan

4 Mizusawa VLBI Observatory, National Astronomical Observatory of Japan, Hoshigaoka2-12, Mizusawa-ku, Oshu, Iwate 023-0861, Japan

${ }^{5}$ Graduate School of Science and Engineering, Yamaguchi University, Yoshida 1677-1, Yamaguchi, Yamaguchi 753-8511, Japan

6 The Research Institute for Time Studies, Yamaguchi University, Yoshida 1677-1, Yamaguchi, Yamaguchi 753-8511, Japan

${ }^{7}$ Korea Astronomy and Space Science Institute, Hwaam-dong 61-1, Yuseong-gu, Daejeon, 305-348, Republic of Korea

8 Joint Institute for VLBI in Europe, Postbus 2, 7990 AA, Dwingeloo, The Netherlands

${ }^{9}$ Department of Astronomical Sciences, SOKENDAI (The Graduate University for Advanced Studies), Hoshigaoka2-12, Mizusawa-ku, Oshu, Iwate 023-0861, Japan a driving source of a so-called low velocity $\left(18 \mathrm{~km} \mathrm{~s}^{-1}\right)$ bipolar outflow along the northeast-southwest (NE-SW) direction with a scale of $1000 \mathrm{AU}$ traced by the thermal SiO lines (Plambeck et al. 2009; Zapata et al. 2012; Niederhofer et al. 2012; Greenhill et al. 2013). At the position of the central YSO, vibrationally excited $\mathrm{SiO}$ masers are detected, which is a quite rare case for starforming regions (Zapata et al. 2009). High resolution VLBI observations reveal that the $\mathrm{SiO}$ masers trace a magneto-centrifugal disk wind from the surface of the circumstellar disk with 100 au scale (Matthews et al. 2010). Vibrationally excited molecular lines such as $\mathrm{H}_{2} \mathrm{O}$, $\mathrm{SiO}$, $\mathrm{CO}$, and SO at the excitation energies of 500-3500 K are also detected around Source I by the recent Atacama Large Millimeter/Submillimeter Array (ALMA) observations suggesting a hot molecular gas in the rotating disk and base of the outflow Hirota et al. 2012, 2014, 2016; Plambeck \& Wright 2016).

Continuum emissions are detected from centimeter to submillimeter wavelengths up to $690 \mathrm{GHz}$ (Plambeck \& Wright 2016, for the latest review). Recent high resolution observations with the Very Large Array (VLA) and ALMA resolve the structure of the continuum emission with the deconvolved size of $\sim 100$ au scale (Reid et al. 2007; Goddi et al. 2011; Plambeck et al. 2013; Plambeck \& Wright 2016). Because the continuum source is elongated perpendicular to the low-velocity NE-SW outflow, the continuum emission is thought to trace an edge-on circumstellar disk rather than a radio jet. Possible mechanism of the continuum emission is proposed such as thermal dust gray-body radiation, $\mathrm{H}^{-}$free-free radiation, proton-electron free-free radiation, and their combination (Beuther et al. 2004, 2006; Reid et al. 2007; Plambeck et al. 2013; Hirota et al. 2015; Plambeck \& Wright 2016). The spectral energy distribution (SED) of Source I shows a power-law function with an index of 2 below $350 \mathrm{GHz}$ while it be- 
comes slightly steeper at higher frequency than $350 \mathrm{GHz}$ (Plambeck \& Wright 2016). Such a characteristics along with the lack of turnover in the SED (Hirota et al. 2015) strongly implies the optically thick thermal dust emission (Plambeck \& Wright 2016). The brightness temperature of $500 \mathrm{~K}$ in the submillimeter bands also support an explanation via the thermal dust emission (Plambeck \& Wright 2016).

In this letter, we present the new continuum measurement results with ALMA at band $8(430 \mathrm{GHz}, 460 \mathrm{GHz}$, and $490 \mathrm{GHz}$ ) at a resolution of 100 mas or 40 au. Our data achieve the highest spatial resolution in the recent millimeter/submillimeter observations except that from VLA (Reid et al. 2007; Goddi et al. 2011), and hence, they are helpful to confirm the recent observational results (Hirota et al. 2015; Plambeck \& Wright 2016). Accurate measurements of the continuum flux densities can also constrain a shape of the SED such as a possible signature of excess emission and turnover frequency (e.g. Beuther et al. 2004, 2006; Plambeck et al. 2013; Hirota et al. 2015). Our results support the conclusions obtained from the latest ALMA observations at $350 \mathrm{GHz}$ and $660 \mathrm{GHz}$ (Plambeck \& Wright 2016). The present newer and higher resolution data could put better constraints on the width of the minor axis, and establish an improved lower limit on the brightness temperature.

\section{OBSERVATIONS AND DATA ANALYSIS}

Observations were carried out in 2015 with ALMA as one of the science projects in cycle 2 (2013.1.00048). The tracking center position was $\quad \operatorname{RA}(J 2000)=05 h 35 \mathrm{~m} 14.512 \mathrm{~s}, \quad \operatorname{Decl}(\mathrm{J} 2000)=-$ 05d22'30.57". Details of observations are listed in Table [1] A primary flux calibrator and band-pass calibrator were J0423-013, and J0522-3627, respectively. Secondary gain calibrators were J0607-0834 for observations at $460 \mathrm{GHz}$ and $490 \mathrm{GHz}$ and J0501-0159 at $430 \mathrm{GHz}$. The ALMA correlator provided the four spectral windows with the total bandwidth of $1875 \mathrm{MHz}$ and 937.5 MHz for two windows each. The channel spacings of spectrometers were $976.562 \mathrm{kHz}$ and $488.281 \mathrm{kHz}$ for the spectral windows with the $1875 \mathrm{MHz}$ and $937.5 \mathrm{MHz}$ bandwidths, respectively. Dual polarization data were obtained simultaneously for all the frequency bands.

The data were calibrated and imaged with the Common Astronomy Software Applications (CASA) package. We employed the calibrated data delivered by the EastAsia ALMA Regional Center (EA-ARC). First, visibility data were separated into spectral lines and continuum emissions by setting the line free channels with the CASA task uvcontsub. Next, both phase and amplitude self-calibration was done with the continuum emission of Source I by integrating over all the channels using CASA tasks clean and gaincal. Because of calibration problems in the delivered data due to the large atmospheric opacity around $487 \mathrm{GHz}$, we used only three spectral windows among four ALMA basebands to make a continuum image at $490 \mathrm{GHz}$. For the intensity maps of the continuum emissions, we employed the visibility data with the UV distance longer than $400 \mathrm{k} \lambda$ to resolve out extended emission components (Hirota et al. 2015). The procedure provides an angular resolution of $\sim 100$ mas, corresponding to the linear resolution of $40 \mathrm{au}$ at the distance to Orion KL. We note that the observed peak positions of the continuum emissions at different frequencies are offset from each others as summarized in Table 2. It is probably due to astrometric calibration errors in the ALMA observations/data analysis. Thus, all the maps are registered based on the continuum peak positions, and we will not discuss about absolute position of each emission.

\section{RESULTS AND DISCUSSION}

Figures 1 shows continuum images of Source I at three observed frequencies in ALMA band 8. The source properties are determined by fitting the two dimensional Gaussian to the images and the derived parameters are listed in Table 2. The continuum maps have the sizes of about (230-240) mas $\times(90-110)$ mas with the position angle of 140 degrees. The structure is elongated along the NW-SE direction, which is perpendicular to the lowvelocity NE-SW bipolar outflow. The deconvolved size is 220 mas $\times 50$ mas, corresponding to $90 \mathrm{au} \times 20 \mathrm{au}$ at the distance of Orion KL. All the results are consistent with those derived from previous ALMA observations at $350 \mathrm{GHz}$ and $660 \mathrm{GHz}$ with slightly larger beam size (Plambeck \& Wright 2016).

The flux densities are plotted in Figure 2 along with previous observations (Plambeck et al. 2013; Hirota et al. 2015; Plambeck \& Wright 2016, and references therein). Recent ALMA observations of continuum emissions at $350 \mathrm{GHz}$ and $660 \mathrm{GHz}$ show the optically thick spectral energy distribution (SED) with a power-law index of 2.0 (Plambeck \& Wright 2016). Our measured flux densities agrees well with those of their interpolation.

Because we used only visibilities with the longer baseline length than $400 \mathrm{k} \lambda$ in the imaging, some of the emission could be resolved out. Using the CASA task simobserve, we simulated the ALMA imaging of a Gaussian source model with the size of 220 mas $\times 50$ mas and total flux density of $1 \mathrm{Jy}$. When we assume the same uv coverage of our observation at $460 \mathrm{GHz}$ with a uniform weighting of only $>400 \mathrm{k} \lambda$ data, the derived flux density and deconvolved source size, $0.9996 \mathrm{Jy}$ and 220.0 mas $\times 49.8$ mas can reconstruct the model parameters. Thus, our imaging results provide reliable source size and flux density.

We reanalyze the SED by employing our new data at $460 \mathrm{GHz}$ band. The same fitting procedures are applied to the present data as described in Hirota et al. (2015). If there are more than two observed results previously reported at the same frequency band, we only employ the flux density data with the highest spatial resolution at each band. For our ALMA band 8 results, we only use the $460 \mathrm{GHz}$ data and do not include the $430 \mathrm{GHz}$ and $490 \mathrm{GHz}$ data in order not to put too much weight for our band 8 data in the fitting. When all the data from 6 to $660 \mathrm{GHz}$ are used in the fitting as shown in Figure 2(a), the best fit power-law SED, $F_{\nu}=p \nu^{q}$, has an spectral in$\operatorname{dex} q$ of $1.86 \pm 0.05$. Compared with the best fit SED, the black-body SED with the fixed index of 2.0 shows significant deviation at the lower frequency data below $10 \mathrm{GHz}$ because of the excess emission from the free-free radiation as proposed by Plambeck \& Wright (2016). Alternatively, it may also suggest an unresolved spatial structure of inhomogeneous gas distribution or physical properties such as density/temperature distribution (Beuther et al. 
2004, 2006; Plambeck et al. 2013).

For comparison, we plot the SED at the frequency higher than $100 \mathrm{GHz}$ in Figure 2(b). We cannot see a clear signature of a turnover in the SED that would suggest an optically thin $\mathrm{H}^{-}$free-free or proton-electron freefree radiation (Beuther et al. 2004, 2006; Hirota et al. 2015). The best-fit SED model by using only the high frequencies from $230 \mathrm{GHz}$ to $660 \mathrm{GHz}$ gives the power-law index of 2.16 \pm 0.06 . In all cases, the SED at our band 8 data is consistent with the optically thick black-body radiation. We note that the best-fit power-law index of 2.16 is marginally larger than 2.0. This is consistent with the excess flux at higher frequency than $600 \mathrm{GHz}$ where the dust emission has the larger source size or becomes hotter as already reported by Plambeck \& Wright (2016).

Because the source structure is marginally resolved in the present observations, we can derive the brightness temperature of the continuum emission, as listed in Table2. The brightness temperature of $700-800 \mathrm{~K}$ is slightly higher than that derived from the $350 \mathrm{GHz}$ and $660 \mathrm{GHz}$ continuum data of $500 \mathrm{~K}$ (Plambeck \& Wright 2016). It is bacause our higher resolution data can better constrain the source size, in particular for the minor axis of the edge-on disk. According to Plambeck \& Wright (2016), proton-electron free-free emission is unlikely to be an opacity source because of the lack of hydrogen recombination line and extremely large luminosity required for the optically thick SED. The optically thick $\mathrm{H}^{-}$freefree radiation is also ruled out since unrealistically high density or large disk mass is necessary to satisfy the optically thick SED without a turnover (Plambeck \& Wright 2016). Our new SED fitting with the beam averaged brightness temperature of $700-800 \mathrm{~K}$ agrees with their interpretation via the optically thick thermal dust emission (Plambeck \& Wright 2016).

Nevertheless, there may be a smaller scale structure unresolved with the ALMA beam $\sim 40$ au which could have a higher brightness temperature. In fact, the higher spatial resolution data at $43 \mathrm{GHz}$ observed with VLA show the more compact structure than the present
ALMA beam size with the higher brightness temperature of $1600 \mathrm{~K}$ (Reid et al. 2007). The excess flux densities below $43 \mathrm{GHz}$ is attributed to a contribution from the hotter free-free radiation (Plambeck \& Wright 2016). If the actual disk size or the beam filling factor is smaller by a factor of two than that of the deconvolved size, the brightness temperature becomes higher than $1600 \mathrm{~K}$ observed with the VLA. In this case, we cannot fully rule out a possibility of the $\mathrm{H}^{-}$free-free radiation as an opacity source of the emission. The hydrogen density to achieve the optically thick $\mathrm{H}^{-}$free-free emission up to $660 \mathrm{GHz}$ requires $\sim 10^{12}-10^{13} \mathrm{~cm}^{-3}$ corresponding to a disk mass of $2 M_{\odot}$ (e.g. Reid et al. 2007). The highest resolution imaging that can be achieved with ALMA $<10$ au will be able to resolve a vertical and internal clumpy structure of this edge-on disk for the first time, which will be the key issue to understanding of the basic nature of Source I.

We would like to thank Richard L. Plambeck for valuable comments. This paper makes use of the following ALMA data: ADS/JAO.ALMA\#2013.1.00048.S. ALMA is a partnership of ESO (representing its member states), NSF (USA) and NINS (Japan), together with NRC (Canada), NSC and ASIAA (Taiwan), and KASI (Republic of Korea), in cooperation with the Republic of Chile. The Joint ALMA Observatory is operated by ESO, AUI/NRAO and NAOJ. We thank the staff at ALMA for making observations and reducing the science verification data. T.H. is supported by the MEXT/JSPS KAKENHI Grant Numbers 21224002, 24684011, 25108005, and 15H03646, and the ALMA Japan Research Grant of NAOJ Chile Observatory, NAOJ-ALMA-0006, 0028, 0066. M.H. is supported by the MEXT/JSPS KAKENHI Grant Numbers 24540242 and 25120007. Data analysis were in part carried out on common use data analysis computer system at the Astronomy Data Center, ADC, of the National Astronomical Observatory of Japan.

Facilities: ALMA.

\section{REFERENCES}

Beuther, H., Zhang, Q., Greenhill, L. J., et al. 2004, ApJL, 616, L31

Beuther, H., Zhang, Q., Reid, M. J., et al. 2006, ApJ, 636, 323

Forbrich, J., Rivilla, V. M., Menten, K. M., et al. 2016, ApJ, 822, 93

Garay, G., Moran, J. M., \& Reid, M. J. 1987, 314, 535

Goddi, C., Humphreys, E. M. L., Greenhill, L. J., Chandler, C. J., \& Matthews, L. D. 2011, ApJ, 728, 15

Greenhill, L. J., Goddi, C., Chandler, C. J., Matthews, L. D., \& Humphreys, E. M. L. 2013, ApJ, 770, 32

Hirota, T, Kim, M. K., \& Honma, M. 2012, ApJL, 757, L1

Hirota, T., Kim, M. K., \& Honma, M. 2016, ApJ., 817, 168

Hirota, T., Kim, M. K., Kurono, Y., \& Honma, M. 2014, ApJL, $782, \mathrm{~L} 28$

Hirota, T., Kim, M. K., Kurono, Y., \& Honma, M. 2015, ApJ., 801,82

Kim, M. K., Hirota, T., Honma, M., et al. 2008, PASJ, 60, 991

Kleinmann, D. E. \& Low, F. J. 1967, ApJL, 149, L1

Matthews, L. D., Greenhill, L. J., Goddi, C., et al. 2010, ApJ, 708,80

Menten, K. M. \& Reid, M. J. 1995, ApJL, 445, L157

Menten, K.M., Reid,M. J., Forbrich, J., \& Brunthaler, A. 2007, A\&A, 474, 515
Niederhofer, F. , Humphreys, E. M. L., \& Goddi, C. 2012a, A\&A, 548, A69

Plambeck, R. L., Bolatto, A. D., Carpenter, J. M., et al. 2013, ApJ, 765, 40

Plambeck, R. L., Wright, M. C. H., Friedel, D. N., et al. 2009, ApJL, 704, L25

Plambeck, R. L. \& Wright, M. C. H. 2016, ApJ, in press (arXiv:1608.00996)

Reid, M. J., Menten, K. M., Greenhill, L. J., \& Chandler, C. J. 2007, ApJ, 664, 950

Rivilla, V. M., Chandler, C. J., Sanz-Forcada, J., et al. 2015ApJ, 808,146

Zapata, L. A., Rodríguez, L. F., Kurtz, S. E., O'Dell, C. R. 2004, AJ, 127, 2252

Zapata, L. A., Menten, K., Reid, M., \& Beuther, H. 2009, ApJ, 691,332

Zapata, L. A., Rodríguez, L. F., Schmid-Burgk, J., et al. 2012, ApJL, 745, L17 
TABLE 1

SumMary of OBSERVATIONS

\begin{tabular}{|c|c|c|c|c|c|c|c|c|c|}
\hline $\begin{array}{c}\text { Center } \\
\text { Frequency } \\
(\mathrm{GHz})\end{array}$ & $\begin{array}{c}\text { Date } \\
\text { (in 2015) }\end{array}$ & $\begin{array}{c}\text { Total } \\
\text { bandwidth } \\
(\mathrm{MHz})\end{array}$ & $\begin{array}{l}\text { Number } \\
\text { of } \\
\text { Antennas }\end{array}$ & $\begin{array}{l}\text { Longest } \\
\text { Baseline } \\
(\mathrm{m})\end{array}$ & $\begin{array}{l}\text { On-source } \\
\text { Time } \\
(\mathrm{sec})\end{array}$ & $\begin{array}{c}\text { Median } \\
T_{\text {sys }} \\
(\mathrm{K})\end{array}$ & $\begin{array}{c}\text { beam size } \\
\text { FWHM } \\
(\mathrm{mas} \times \text { mas })\end{array}$ & $\begin{array}{c}\text { PA } \\
\text { (degree) }\end{array}$ & $\begin{array}{c}\mathrm{rms} \\
\left(\mathrm{mJy} \mathrm{beam}^{-1}\right)\end{array}$ \\
\hline 430 & Sep. 22 & 5625 & 35 & 2270 & 1929 & 1200 & $83 \times 67$ & -79.8 & 2.7 \\
\hline 460 & Aug. 27 & 5625 & 40 & 1574 & 410 & 430 & $104 \times 87$ & 82.9 & 4.1 \\
\hline 490 & Jul. 27 & 4688 & 41 & 1466 & 315 & 400 & $111 \times 79$ & 84.5 & 3.8 \\
\hline
\end{tabular}

${ }^{a}$ Center frequencies for LSB (lower side band) and USB (upper side band).
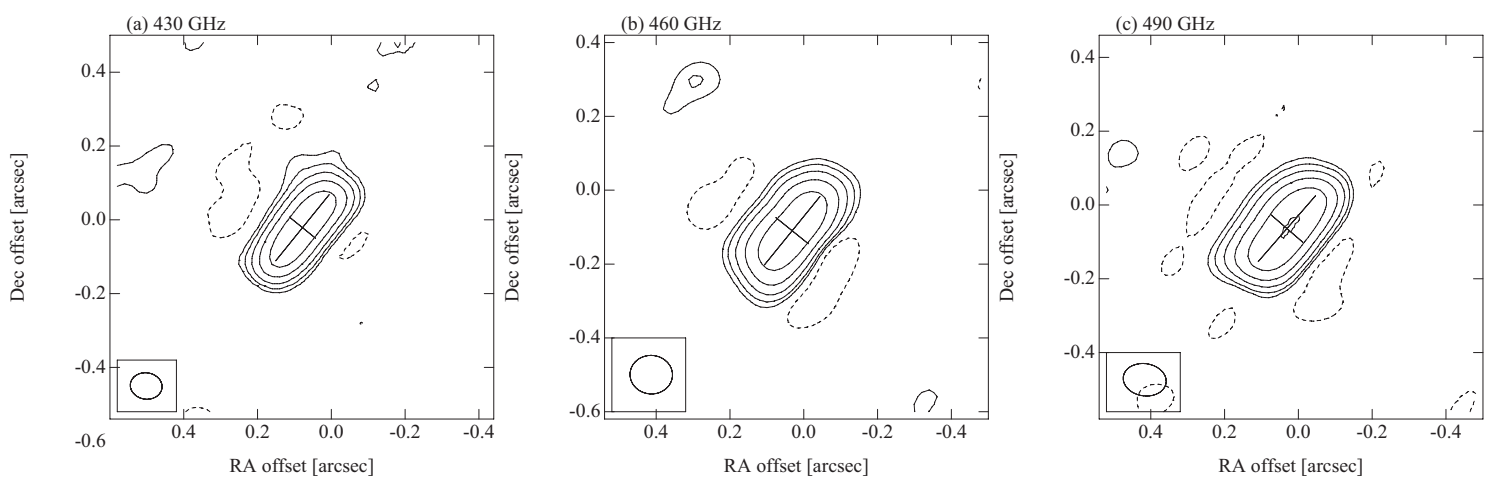

Fig. 1. - Continuum emission maps for band 8 data. Synthesized beam size is indicated at the bottom-left corner in each panel. The contour levels are $-4,4,8,16,32, \ldots \times$ the rms noise level. The $(0,0)$ position is the tracking center position, $\mathrm{RA}(\mathrm{J} 2000)=05 \mathrm{~h} 35 \mathrm{~m} 14.512 \mathrm{~s}$, $\operatorname{Decl}(\mathrm{J} 2000)=-05 \mathrm{~d} 22^{\prime} 30.57^{\prime \prime}$. (a) $430 \mathrm{GHz}$. The noise level $(1 \sigma)$ and peak intensity are $2.7 \mathrm{mJy}$ beam ${ }^{-1}$ and 324 mJy beam ${ }^{-1}$, respectively. (b) $460 \mathrm{GHz}$. The noise level $(1 \sigma)$ and peak intensity are $4.1 \mathrm{mJy}^{\text {beam }}{ }^{-1}$ and $481 \mathrm{mJy}$ beam $^{-1}$, respectively. (c) $490 \mathrm{GHz}$. The noise level $(1 \sigma)$ and peak intensity are $3.8 \mathrm{mJy}$ beam $^{-1}$ and $506 \mathrm{mJy}^{\text {beam }}{ }^{-1}$, respectively. A cross in each panel represents the major and minor axis of the image. Note that the positions are offset from each other (see text).

TABLE 2

Gauss FitTing Results

\begin{tabular}{|c|c|c|c|c|c|c|c|c|c|}
\hline $\begin{array}{l}\text { Frequency } \\
\quad(\mathrm{GHz})\end{array}$ & $\begin{array}{c}\Delta \alpha^{\mathrm{a}} \\
(\mathrm{mas})\end{array}$ & $\begin{array}{c}\Delta \delta^{\mathrm{a}} \\
(\mathrm{mas})\end{array}$ & $\begin{array}{l}\text { Convolved size } \\
(\text { mas } \times \text { mas })\end{array}$ & $\begin{array}{l}\text { PA } \\
\text { (deg) }\end{array}$ & $\begin{array}{l}\text { Deconvolved size } \\
\quad(\text { mas } \times \text { mas })\end{array}$ & $\begin{array}{l}\text { PA } \\
\text { (deg) }\end{array}$ & $\begin{array}{l}\text { Peak intensity } \\
\left(\mathrm{mJy}^{-} \text {beam }^{-1}\right)\end{array}$ & $\begin{array}{l}\text { Integrated flux } \\
(\mathrm{mJy})\end{array}$ & $\begin{array}{c}T_{b} \\
(\mathrm{~K})\end{array}$ \\
\hline 440 & $78.5(5)$ & $-21.5(5)$ & $229.6(16) \times 91.4(6)$ & $141.4(3)$ & $216.7(16) \times 53.0(12)$ & $143.1(3)$ & $353(2)$ & $1338(11)$ & $735(6)$ \\
\hline 460 & $31.7(5)$ & $-109.7(6)$ & $238.8(16) \times 112.6(8)$ & $141.3(3)$ & $220.4(18) \times 52.3(18)$ & $143.1(4)$ & $520(4)$ & $1547(14)$ & $777(7)$ \\
\hline 490 & $31.0(4)$ & $-64.0(5)$ & $236.7(13) \times 113.9(6)$ & $138.8(3)$ & $218.9(15) \times 50.9(17)$ & $142.4(3)$ & $557(3)$ & $1709(12)$ & $756(6)$ \\
\hline
\end{tabular}

Note. - Numbers in parenthesis represent fitting errors in unit of the last significant digits.

a Offset from the tracking center position. 

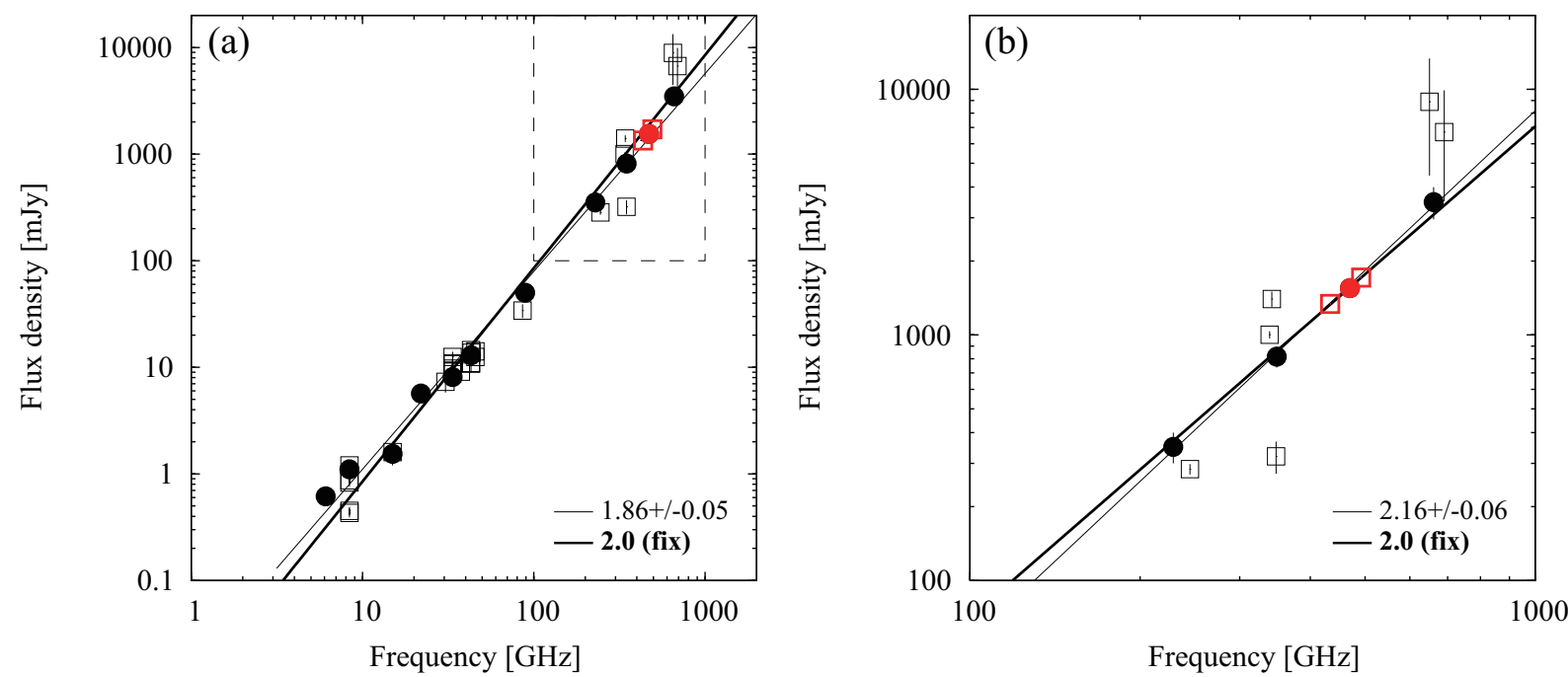

FIG. 2.- Spectral energy distribution (SED) of Source I. (a) All the frequency bands from 6 to 660 GHz. (b) Frequency bands enlarged from 100-1000 GHz as indicated by a dashed box in panel (a). Bold solid lines indicate the black-body SED with the fixed power-law index of 2.0. Thin solid lines show the best-fit single power-law models $F_{\nu}=p \nu^{q}$. The derived power-law indexes are shown in each panel. Red open and filled circles show our ALMA band 8 data at $430 \mathrm{GHz}, 460 \mathrm{GHz}$, and $490 \mathrm{GHz}$. Black open squares and filled circles represent all the data including the present ALMA results (Plambeck et al. 2013; Hirota et al. 2015; Plambeck \& Wright 2016, and references therein), In the SED fitting, we only employed the flux density data obtained with the highest spatial resolution in each band, as indicated by filled black and red circles. 